\title{
FUNCTIONAL DIFFERENTIAL EQUATIONS WITH NONLOCAL INITIAL CONDITIONS
}

\author{
SERGIU AIZICOVICI and YUN GAO \\ Ohio University, Department of Mathematics \\ Athens, $\mathrm{OH} \quad 45701$ USA
}

(Received January, 1996; Revised May, 1996)

\begin{abstract}
We study the existence, uniqueness, asymptotic properties, and continuous dependence upon data of solutions to a class of abstract nonlocal Cauchy problems. The approach we use is based on the theory of $m$-accretive operators and related evolution equations in Banach spaces.
\end{abstract}

Key words: Functional Differential Equation, m-Accretive Operator, Nonlocal Cauchy Problem.

AMS subject classifications: $34 \mathrm{G} 20,34 \mathrm{~K} 30,45 \mathrm{~N} 05$.

\section{Introduction}

This paper is concerned with the nonlocal Cauchy problem

$$
\begin{gathered}
u^{\prime}(t)+A u(t)+G(u)(t) \ni 0, \quad 0<t<T \\
u(0)=g(u),
\end{gathered}
$$

in a Banach space $X$. Here $A$ is an $m$-accretive (possibly multivalued) operator in $X, G: C([0, T] ; X) \rightarrow L^{1}(0, T ; X)$, and $g: C([0, T] ; X) \rightarrow X$. Points of interest are the existence, uniqueness, regularity, dependence upon data, and asymptotic properties (as $T \rightarrow \infty$ ) of solutions of (1.1).

Much of the motivation for our study of (1.1) lies in the fact that we thereby generalize result due to several other authors. Byszewski [5] proves the existence and uniqueness of solutions to

$$
\begin{gathered}
u^{\prime}(t)+A u(t)=f(t, u(t)), \quad 0<t<T \\
u(0)+g\left(t_{1}, \ldots, t_{p}, u\left(t_{1}\right), \ldots, u\left(t_{p}\right)\right)=u_{0},
\end{gathered}
$$

where $0<t_{1}<\ldots<t_{p} \leq T$ are fixed, $u_{0} \in X,-A$ is the infinitesimal generator of a linear $C_{0}$ semigroup on $X, f:[0, T] \times X \rightarrow X$ satisfies a Lipschitz condition in its second variable, and $g:[0, T]^{p} \times X^{p} \rightarrow X$ yields a Lipschitz continuous map from $C([0, T] ; X)$ into $X$. (A slightly more general nonlocal condition appears in [7].) 
Balachandran and Ilamaran [2] study (1.2) with $f(t, u(\sigma(t)))$ in place of $f(t, u(t))$, where $\sigma:[0, T] \rightarrow[0, T]$ is absolutely continuous and $f:[0, T] \times X \rightarrow X$. In [6], Byszewski discusses only the uniqueness of strong solutions to the nonlinear problem

$$
\begin{gathered}
u^{\prime}(t) \in A u(t), \quad 0<t<T \\
u(0)+g(u)=u_{0},
\end{gathered}
$$

under the assumption that $A: X \rightarrow 2^{X}$ is dissipative, $u_{0} \in X$, and $g$ is a contraction from $C([0, T] ; X)$ into $X$. Lin and Liu [17] consider the semilinear integrodifferential equation

$$
u^{\prime}(t)=A\left[u(t)+\int_{0}^{t} F(t-s) u(s) d s\right]+f(t, u(t)), \quad 0<t<T,
$$

together with the same initial condition as the one in (1.2), where $A$ generates a linear strongly continuous semigroup on $X, F(t)$ is a bounded linear operator on $X$ for all $t \in[0, T]$, and $f:[0, T] \times X \rightarrow X$. Concrete nonlocal initial-boundary value problems for semilinear parabolic equations arising in physics (particularly in the mathematical modeling of heat conduction or diffusion processes) are analyzed in $[10$, $13,16]$.

The present work may be viewed as an attempt to develop a general theory for abstract problems of the type (1.1). The existing studies of (1.2), (1.3) and (1.4) are extended in various directions. Our basic tools are methods and results for differential equations governed by $m$-accretive operators in Banach spaces, as well as fixed point techniques.

The outline of the paper is the following. In Section 2 we recall some facts about $m$-accretive operators and nonlinear evolution equations. Sections 3 and 4 contain the main existence, uniqueness, and continuous dependence results. The asymptotic properties of solutions of (1.1) are studied in Section 5, while Section 6 deals with the case when $A$ depends on time. A model nonlocal integro-partial differential equation is presented in Section 7.

\section{Preliminaries}

For further background and details of this section we refer the reader to [8].

Let $X$ be a real Banach space of norm $|\cdot|$. A set-valued operator $A$ in $X$ with domain $D(A)$ and range $R(A)$, is said to be accretive if $\left|x_{1}-x_{2}\right| \leq\left|x_{1}-x_{2}+\lambda\left(y_{1}-y_{1}\right)\right|$, for all $\lambda>0$ and $y_{i} \in A x_{i}, i=1,2 . A$ is called $m$-accretive if it is accretive and $R(I+\lambda A)=X$, for all $\lambda>0$. (Here $I$ stands for the identity on $X$.) The Yosida approximation $A_{\lambda}(\lambda>0)$ of an $m$-accretive operator $A$ is given by $A_{\lambda}=\lambda^{-1}\left(I-J_{\lambda}\right)$, where $J_{\lambda}=(I+\lambda A)^{-1}$ is the resolvent of $A$. Accretivity can also be formulated in terms of the mapping $[\cdot, \cdot]: X \times X \rightarrow \mathbb{R}$, defined by

$$
[y, x]=\lim _{\lambda \downarrow 0} \lambda^{-1}(|x+\lambda y|-|x|), \forall x, y \in X .
$$

Then $A$ is accretive if and only if $\left[y_{2}-y_{1}, x_{2}-x_{1}\right] \geq 0, \forall y_{i} \in A x_{i}, \quad i=1,2$. In the case when $X$ is a Hilbert space, $m$-accretivity is equivalent to maximal monotonicity. See $[3,4]$ for more details. An important subclass of maximal monotone operators is that of sub-differentials. If $\varphi$ is a proper, convex, and lower semicontinuous (l.s.c.) function from the Hilbert space $X$ into $(-\infty,+\infty]$, then its subdifferential $\partial \varphi$ is 
given by

$$
\partial \varphi(x)=\{y \in X: \varphi(z)-\varphi(x) \geq(y, z-x), \forall z \in D(\varphi)\}
$$

where $D(\varphi)=\{x \in X: \varphi(x)<\infty\}$, and $($,$) denotes the inner product in X$.

Next consider the Cauchy problem

$$
\begin{gathered}
u^{\prime}(t)+A u(t) \ni f(t), \quad 0<t<T \\
u(0)=u_{0},
\end{gathered}
$$

where $A$ is $m$-accretive (or $A+\omega I$ is $m$-accretive for some $\omega \in \mathbb{R}$ ) in $X, f \in L^{1}(0, T ; X)$ and $u_{0} \in \overline{D(A)}$. It is well-known that $(2.1)$ has a unique mild solution $u \in C([0, T] ; \overline{D(A))}$, which is the uniform limit of a sequence of approximate solutions. (Each approximate solution is a piecewise constant function which is defined recursively by means of the resolvent of $A$; see [8] for a precise definition.) If $u$ and $\widehat{u}$ are respectively mild solutions of $u^{\prime}+A u \ni f$ and $\widehat{u}^{\prime}+A \widehat{u} \ni \widehat{f}$, with $A m$ accretive, then

$$
|u(t)-\widehat{u}(t)| \leq|u(0)-\widehat{u}(0)|+\int_{0}^{t}|f(s)-\widehat{f}(s)| d s, \quad \forall t \in[0, T] .
$$

In the case $A+\omega I$ is $m$-accretive $(\omega \neq 0),(2.2)$ changes to

$$
|u(t)-\widehat{u}(t)| \leq e^{\omega t}|u(0)-\widehat{u}(0)|+\int_{0}^{t} e^{\omega(t-s)}|f(s)-\widehat{f}(s)| d s, \quad 0 \leq t \leq T .
$$

\section{Existence Results}

We consider the nonlocal initial-value problem (1.1) under the following basic assumptions:

(H1) $A$ is $m$-accretive on $X$,

$(H 2) \quad G: C\left([0, T] ; \overline{D(A))} \rightarrow L^{1}(0, T ; X)\right.$ satisfies

$$
\|G(u)-G(v)\|_{L^{1}(0, T ; X)} \leq M\|u-v\|_{C([0, T] ; X)}, \quad \forall u, v \in C([0, T] ; \overline{D(A))}
$$

for some $M>0$,

$g: C([0, T] ; \overline{D(A))} \rightarrow \overline{D(A)}$ is such that

$$
|g(u)-g(v)| \leq m\|u-v\|_{C([0, T] ; X)}, \quad \forall u, v \in C([0, T] ; \overline{D(A))}
$$

with $m>0$.

Definition 3.1: A function $u \in C([0, T] ; \overline{D(A))}$ is said to be a mild solution of (1.1) if it is a mild solution of (2.1) with $-G(u)$ and $g(u)$ in place of $f$ and $u_{0}$, respectively.

Definition 3.2: A strong solution of (1.1) on $[0, T]$ is a function $u \in$ $W^{1,1}(0, T ; X) \cap C\left([0, T] ; \overline{D(A))}\right.$ satisfying $u(0)=g(u)$ and $u^{\prime}(t)+A u(t)+G(u)(t) \ni$ 0 , a.e. on $(0, T)$.

Our first result establishes the existence and uniqueness of mild solutions to (1.1).

Theorem 3.3: Let $(H 1),(H 2)$ and $(H 3)$ hold. In addition, assume that 


$$
m+M<1
$$

Then problem (1.1) has a unique mild solution.

Proof: Let $v \in C([0, T] ; \overline{D(A))}$ be fixed, and consider $(2.1)$ with $-G(v)$ and $g(v)$ in place of $f$ and $u_{0}$, respectively. Denote the corresponding mild solution by $J v$. We have thereby defined a mapping $J$ of $C([0, T] ; \overline{D(A))}$ into itself. By (2.2), we obtain

$$
|(J v)(t)-(J w)(t)| \leq|g(v)-g(w)|+\int_{0}^{t}|G(v)(s)-G(w)(s)| d s
$$

for all $0 \leq t \leq T$, and $v, w \in C([0, T] ; \overline{D(A))}$. Using $(H 2)$ and $(H 3)$ in (3.2) yields

$$
\|J v-J w\|_{C([0, T] ; X)} \leq(m+M)\|v-w\|_{C([0, T] ; X)},
$$

for any $v, w \in C([0, T] ; \overline{D(A))}$. In view of $(3.1)$, this implies that $J$ is a strict contraction on $C([0, T] ; \overline{D(A))}$. The unique fixed point of $J$ is obviously the desired mild solution of (1.1) (cf. Definition 3.1). The proof is complete.

If in place of $(H 1)$ we assume

$\left(H 1^{\prime}\right) \quad A+\omega I$ is $m$-accretive for some $\omega>0$,

then the conclusion of Theorem 3.3 holds provided that condition (3.1) is changed to

$$
m+M<e^{-\omega T} \text {. }
$$

An obvious modification in the proof of Theorem 3.3 (use (2.3) in place of (2.2)) leads to:

Theorem 3.4: Let $\left(H 1^{\prime}\right),(H 2),(H 3)$ and (3.3) be satisfied. Then (1.1) has a unique mild solution.

Condition (3.1) can be weakened in the case when $G$ is a Volterra type operator. Specifically, let $G: C\left([0, T]: \overline{D(A))} \rightarrow L^{1}(0, T ; X)\right.$ satisfy

$$
\|G(u)-G(v)\|_{L^{1}(0, t ; X)} \leq \int_{0}^{t} \gamma(s)\|u-v\|_{L^{\infty}(0, s ; X)} d s, \quad 0 \leq t \leq T,
$$

for all $u, v \in C\left([0, T] ; \overline{D(A))}\right.$, with $\gamma \in L^{1}(0, T), \gamma \geq 0$.

In addition, assume

$$
\int_{0}^{t}[G(u)(s)-G(v)(s), u(s)-v(s)] d s \geq 0,
$$

for any $t \in[0, T]$, and $u, v \in C([0, T] ; \overline{D(A))}$.

Remark 3.5: Conditions (3.4) and (3.5) are satisfied if $X$ is a Hilbert space, and $G(u)(t)=\int_{0}^{t} a(t-s) u(s) d s, \quad \forall u \in C([0, T] ; X)$, where $a \in L^{1}(0, T)$ is a kernel of positive type [18].

Theorem 3.6: If (H1), (H3), (3.4), (3.5) hold and $m<1$, then there exists a unique mild solution of (1.1).

Proof: First note that (3.4) implies $(H 2)$ with $M=\int_{0}^{T} \gamma(s) d s$. However, since we do not restrict the 'size' of $\gamma$, condition (3.1) is not satisfied in general, so that Theorem 3.3 does not apply. 
Let $v \in C([0, T] ; \overline{D(A))}$ be fixed, and consider the problem

$$
\begin{gathered}
u^{\prime}(t)+A u(t)+G(u)(t) \ni 0, \quad 0<t<T \\
u(0)=g(v) .
\end{gathered}
$$

Taking into account (3.4), we can invoke Theorem 1 in [9] to conclude that (3.6) has a unique mild solution $u \in C([0, T] ; \overline{D(A))}$. Furthermore, because of $(3.5)$, if $u, \widehat{u}$ are mild solutions of (3.6) corresponding to $v, \widehat{v}$ respectively, then by [14, Remark 3.2],

$$
|u(t)-\widehat{u}(t)| \leq|g(v)-g(\widehat{v})|, \quad 0 \leq t \leq T .
$$

Employing $(H 3)$ (with $m<1$ ) in $(3.7)$ we arrive at the conclusion that the map $\widehat{J}$ that associates with each $v \in C([0, T] ; \overline{D(A))}$ the unique mild solution $u$ of $(3.6)$ is a strict contraction on $C([0, T] ; \overline{D(A))}$. It follows that $\widehat{J}$ has a unique fixed point, which is the mild solution of (1.1). This completes the proof.

We next examine two cases when the mild solutions of (1.1) have greater regularity than mere continuity. (In particular, they are strong solutions, in the sense of Definition 3.2.)

Theorem 3.7: Let $X$ be a Hilbert space and $A=\partial \varphi$ where $\varphi: X \rightarrow(-\infty,+\infty]$ is proper, convex and l.s.c. In addition, assume (H2), (H3), (3.1), and let $u$ be the mild solution of (1.1). If $G(u) \in L^{2}(0, T ; X)$ then $u \in W^{1,2}(\varepsilon, T ; X), \forall 0<\varepsilon<T$, and satisfies $(1.1)_{1}$ a.e. on $(0, T)$, and $(1.1)_{2}$. If also $g(u) \in D(\varphi)$, then $u \in W^{1,2}(0, T ; X)$ is a strong solution of $(1.1)$ on $[0, T]$.

Proof: The existence and uniqueness of $u$ is guaranteed by Theorem 3.3. Notice that $u$ is a mild solution of (2.1) with $f=-G(u)$ and $u_{0}=g(u)$. Apply [4, Theorem 3.6] to conclude.

Theorem 3.8: Let the conditions of Theorem 3.6 be satisfied. In addition, suppose that there is a function $\alpha:[0, \infty) \rightarrow[0, \infty)$ such that

$$
\begin{gathered}
\operatorname{var}(G(u):[0, t]) \leq \alpha(R)(1+\operatorname{var}(u:[0, t]), \quad 0 \leq t \leq T \\
\left|G(u)\left(0^{+}\right)\right| \leq \alpha(R)
\end{gathered}
$$

whenever $u \in C\left([0, T] ; \overline{D(A))}\right.$ is of bounded variation and $\|u\|_{C([0, T] ; X)} \leq R$. Let $u$ be the mild solution of (1.1). If $g(u) \in D(A)$, then $u$ is Lipschitz continuous on $[0, T]$. If also $X$ is reflexive, then $u$ is a strong solution to (1.1).

Proof: Theorem 3.6 yields the existence and uniqueness of $u$. Clearly $u$ may be viewed as a mild solution to (3.6) with $v=u$. Because of (3.8) we may appeal to [9, Theorem 2] to obtain the desired conclusions, provided that, in addition, $u(0)=g(u) \in D(A)$.

\section{A Continuous Dependence Theorem}

Throughout this section we assume that $A, G$, and $g$ satisfy $(H 1),(H 2)$, and $(H 3)$, respectively, and that $(3.1)$ holds. For each $n=1,2, \ldots$, we consider an $m$-accretive operator $A_{n}$ in $X$ and two mappings $G_{n}: C([0, T] ; X) \rightarrow L^{1}(0, T ; X)$, and $g_{n}: C([0, T]$; 
$X) \rightarrow \overline{D\left(A_{n}\right)}$ satisfying the following conditions:

$$
\begin{aligned}
& \lim _{n \rightarrow \infty}\left(I+\lambda A_{n}\right)^{-1} x=(I+\lambda A)^{-1} x, \quad \forall \lambda>0, \quad x \in X, \\
& \left\|G_{n}(u)-G_{n}(v)\right\|_{L^{1}(0, T ; X)} \leq M\|u-v\|_{C([0, T] ; X)},
\end{aligned}
$$

for all $u, v \in C([0, T] ; X)$, with the same constant $M$ as in $(H 2)$,

$$
\begin{gathered}
\lim _{n \rightarrow \infty} G_{n}(u)=G(u) \text { in } L^{1}(0, T ; X), \quad \forall u \in C([0, T] ; \overline{D(A))}, \\
\left|g_{n}(u)-g_{n}(v)\right| \leq m\|u-v\|_{C([0, T] ; X)}, \quad \forall u, v \in C([0, T] ; X),
\end{gathered}
$$

where $m$ is the constant which appears in $(H 3)$,

$$
\lim _{n \rightarrow \infty} g_{n}(u)=g(u), \quad \forall u \in C([0, T] ; \overline{D(A))} .
$$

Let $u$ be the mild solution of (1.1). By virtue of (3.1), (4.2), and (4.4), Theorem 3.3 can also be applied to (1.1) with $A_{n}, G_{n}$ and $g_{n}$ in place of $A, G$ and $g$, respectively to obtain the corresponding mild solutions $u_{n}(n=1,2, \ldots)$.

Theorem 4.1: Let assumptions (H1)-(H3), and (3.1), (4.1)-(4.5) be satisfied. Then,

$$
\lim _{n \rightarrow \infty} u_{n}=u \text { in } C([0, T] X)
$$

Proof: Let $v_{n}$ denote the unique mild solution of

$$
\begin{gathered}
v_{n}^{\prime}(t)+A_{n} v_{n}(t)+G_{n}(u)(t) \ni 0,0<t<T \\
v_{n}(0)=g_{n}(u)
\end{gathered}
$$

where $u$ is the mild solution of (1.1). By (2.2), we have

$$
\begin{gathered}
\left|u_{n}(t)-u(t)\right| \leq\left|u_{n}(t)-v_{n}(t)\right|+\left|v_{n}(t)-u(t)\right| \\
\leq\left|g_{n}\left(u_{n}\right)-g_{n}(u)\right|+\left|v_{n}(t)-u(t)\right|+\int_{0}^{T}\left|G_{n}\left(u_{n}\right)(s)-G_{n}(u)(s)\right| d s,
\end{gathered}
$$

for any $t \in[0, T]$. Taking into account (4.2) and (4.4), we infer from (4.8) that

$$
(1-m-M)\left\|u_{n}-u\right\|_{C([0, T] ; X)} \leq\left\|v_{n}-u\right\|_{C([0, T] ; X)} .
$$

Conditions (4.1), (4.3), and (4.5) enable us to apply Theorem 6 of [8] to (4.7) and conclude that $v_{n} \rightarrow u$ in $C([0, T] ; X)$, as $n \rightarrow \infty$. The latter, (3.1) and (4.9) lead to (4.6). The proof is complete.

\section{Asymptotic Properties}

We discuss problem $(1.1)$ on $[0, \infty)$ rather than on a finite interval. A mild solution to $(1.1)$ on $[0, \infty)$ is a continuous function $u:[0, \infty) \rightarrow X$ satisfying Definition 3.1 for any $T \in(0, \infty)$. We will use $B C([0, \infty) ; X)$ to designate the space of all bounded 
continuous functions from $[0, \infty)$ into $X$; this is a Banach space when equipped with the standard sup-norm.

We assume throughout that ( $H 1)$ holds, and that, in addition,

$$
A^{-1} 0 \neq \emptyset \text {. }
$$

We first modify $(H 2)$ and $(H 3)$ as follows:

$\left(H 2^{\prime}\right) \quad G: B C\left([0, \infty) ; \overline{D(A))} \rightarrow L^{1}(0, \infty ; X)\right.$ is such that

$$
\begin{aligned}
& \|G(u)-G(v)\|_{L^{1}(0, \infty ; X)} \leq M\|u-v\|_{B C([0, \infty) ; X)}, \forall u, v \in B C([0, \infty) ; \overline{D(A))}, \\
& \quad \text { with } M>0, \\
& \left(H 3^{\prime}\right) \quad g: B C([0, \infty) ; \overline{D(A))} \rightarrow \overline{D(A)} \text { satisfies } \\
& \quad|g(u)-g(v)| \leq m\|u-v\|_{B C([0, \infty) ; X)}, \forall u, v \in B C([0, \infty) ; \overline{D(A))}, \\
& \quad \text { for some } m>0 .
\end{aligned}
$$

Remark 5.1: A simple case when $\left(H 2^{\prime}\right)$ is satisfied corresponds to $G(u)(t)=$ $f(t, u(t)), \forall u \in B C([0, \infty) ; X)$, where $f:[0, \infty) \times X \rightarrow X$ is a Carathéodory type function with the property that $f(\cdot, 0) \in L^{1}(0, \infty)$, and $\mid f(t, u)-$ $f(t, v)|\leq \delta(t)| u-v \mid$, for all $t \geq 0, u, v \in X$, where $\delta \geq 0, \delta \in L^{1}(0, \infty)$. As regards $g$, one may take $g(u)=\|\theta\|_{L^{1}(0, \infty)}^{-1} \int_{0}^{\infty} \theta(s) u(s) d s(u \in B C([0, \infty) ; X))$, with $\theta \in$ $L^{1}(0, \infty), \quad\|\theta\|_{L^{1}}>0$. Then $\left(H 3^{\prime}\right)$ is obviously satisfied. Such a condition of integral type appears in [10].

Theorem 5.2: Let $(H 1),\left(H 2^{\prime}\right),\left(H 3^{\prime}\right),(3.1)$, and (5.1) hold. Then (1.1) has a unique mild solution $u \in B C([0, \infty) ; X)$ such that

$$
\lim _{t \rightarrow \infty}|u(t)-x| \text { exists, } \forall x \in A^{-1} 0 .
$$

Proof: For each fixed $v \in B C([0, \infty) ; \overline{D(A))}$, let $w$ be the unique mild solution of $w^{\prime}+A w \ni-G(v), w(0)=g(v)$. Since $G(v) \in L^{1}(0, \infty ; X)$ and (5.1) holds we can use Lemma 3.2 of [14] (take $G \equiv 0$ there) to conclude that $w \in L^{\infty}(0, \infty ; X)$. We thereby define the map $F: B C([0, \infty) ; \overline{D(A))} \rightarrow B C([0, \infty) ; \overline{D(A))}$ by $F v=w$. With the arguments as in the proof of Theorem 3.3 (cf. (3.2)) we deduce by $\left(H 2^{\prime}\right),\left(H 3^{\prime}\right)$, and (3.1) that $F$ is a strict contraction on $B C([0, \infty) ; \overline{D(A))}$. The unique fixed point $u$ of $F$ in $B C([0, \infty) ; \overline{D(A))}$ is the mild solution of $(1.1)$ on $[0, \infty)$. To derive $(5.2)$ we first observe that $u$ is a mild solution to (2.1) with $f=-G(u) \in L^{1}(0, \infty ; X)$ and $u_{0}=g(u)$, and then apply again [14, Lemma 3.2].

We next consider the case in which $G$ is an operator of Volterra type (cf. Theorem 3.6). Specifically, we study the functional differential equation

$$
\begin{gathered}
u^{\prime}(t)+A u(t)+G(u)(t) \ni f(t), \quad t \geq 0 \\
u(0)=g(u)
\end{gathered}
$$

under the following assumptions on $G$ (cf. (3.4), (3.5)):

and

$$
G: C\left([0, \infty) ; \overline{D(A))} \rightarrow L_{\mathrm{loc}}^{1}([0, \infty) ; X)\right.
$$

$$
G: C\left([0, \infty) ; \overline{D(A))} \rightarrow L^{1}(0, T ; X) \text { for all } T>0,\right.
$$


with

$$
G\left(u^{T}\right)=(G(u))^{T}, \quad \forall u \in C([0, \infty) ; \overline{D(A))},
$$

where $u^{T}$ and $(G(u))^{T}$ denote the respective restrictions of $u$ and $G(u)$ on $[0, T]$.

There exists $\gamma \in L_{\text {loc }}^{1}\left([0, \infty) ; \mathbb{R}_{+}\right)$such that

$$
\|G(u)-G(v)\|_{L^{1}(0, t ; X)} \leq \int_{0}^{t} \gamma(s)\|u-v\|_{L^{\infty}(0, s ; X)} d s, \quad t \geq 0
$$

for all $u, v \in C([0, \infty) ; \overline{D(A))}$,

$$
\int_{0}^{t}[G(u)(s)-G(v)(s), u(s)-v(s)] d s \geq 0, \quad t \geq 0,
$$

for any $u, v \in C([0, \infty) ; \overline{D(A))}$.

As regards $f$, we assume that

$$
f \in L^{1}(0, \infty ; X) .
$$

Theorem 5.3: Let (H1), (H3') (with $0<m<1)$, (5.1), and (5.4)-(5.7) be satisfied. Then problem $(5.3)$ has a unique mild solution $u \in B C([0, \infty) ; X)$. If $(5.6)$ is strengthened to $G(v) \in L^{1}(0, \infty ; X)$ for each constant function $v(t) \equiv v \in D(A)$, and

$$
[G(v)(t)-G(w)(t), v(t)-w(t)] \geq \frac{d}{d t}(k *|v-w|(t)), \text { a.e. on }(0, \infty)
$$

for all $v, w \in W_{\text {loc }}^{1,1}([0, \infty) ; X)$, where $k \in L^{1}(0, \infty)$ is nonnegative and nonincreasing, and '*' stands for the convolution product, then (5.2) also holds true.

Proof: For each $v \in B C([0, \infty) ; \overline{D(A))}$ we consider the Cauchy problem

$$
\begin{gathered}
w^{\prime}(t)+A w(t)+G(w)(t) \ni f(t), \quad t \geq 0 \\
w(0)=g(v) .
\end{gathered}
$$

By the results in [9], (5.9) has a unique mild solution $w \in C([0, \infty) ; \overline{D(A))}$. Moreover, in view of (5.1) and (5.6) we may apply [14, Remark 3.1] to conclude that $w \in B C([0, \infty) ; X)$. As in the proof of Theorem 3.6 it then follows (use (3.7) and $\left(H 3^{\prime}\right)$ with $\left.0<m<1\right)$ that the map that associates with each $v \in B C([0, \infty) ; \overline{D(A))}$ the mild solution $w$ of $(5.9)$ is a strict contraction on $B C([0, \infty) ; \overline{D(A))}$. Its unique fixed point $u \in B C([0, \infty) ; X)$ is the desired mild solution of (5.3). If (5.8) holds, then Lemma 3.2 of [14] (when applied to (5.3)) implies (5.2). The proof is complete.

Remark 5.4: A classical example of a mapping $G$ satisfying (5.4), (5.5), and $(5.8)$ is given by $G(u)(t)=\frac{d}{d t}(k * u)(t)=k(0) u(t)+\int_{0}^{t} u(t-s) d k(s), \forall u \in C([0, \infty) ; X)$, where $k:[0, \infty) \rightarrow[0, \infty)$ is nonincreasing and $k \in L^{1}(0, \infty)$. See [12, Lemma 2.7] and [14, Lemma 5.1].

Analogs of other asymptotic results for Volterra equations [1, 12, 14] can also be proved for problems of the form (5.3). For instance, Theorem 3.3 in [14] leads to:

Theorem 5.5: Let $(H 1),\left(H 3^{\prime}\right)$ (with $\left.0<m<1\right)$, (5.1), (5.4), (5.5), (5.7), and (5.8) hold, and let $u$ be the unique mild solution of (5.3). If, in addition, $X$ is reflexive and has a weakly sequentially continuous duality map, then the following 
conditions are equivalent:

(i) weak-lim w $\rightarrow \infty u(t)$ exists,

(ii) weak-lim ${ }_{t \rightarrow \infty} u(t)$ exists and belongs to $A^{-1} 0$,

(iii) $\quad \omega_{w}(u) \subset A^{-1} 0, \quad$ with $\quad \omega_{w}(u)=\left\{y \in X: y=\right.$ weak-lim $m_{n \rightarrow \infty} u\left(t_{n}\right)$, for some $\left.t_{n} \rightarrow \infty\right\}$.

Well-known examples of reflexive Banach spaces with weakly sequentially continuous duality maps are Hilbert spaces and $\ell_{p}$ sequence spaces $(1<p<\infty)$.

\section{The Time Dependent Case}

The existence-uniqueness theory for (1.1) can easily be extended to the case when $A$ depends on time. Consider a family $\{A(t), 0 \leq t \leq T\}$ of operators in $X$ satisfying

For almost every $t \in[0, T], A(t)$ is $m$-accretive.

There exist $\lambda_{0}>0, h \in L^{1}(0, T ; X)$, and a nondecreasing continuous function $L:[0, \infty) \rightarrow[0, \infty)$ such that

$$
\left|A_{\lambda}(t) x-A_{\lambda}(s) x\right| \leq|h(t)-h(s)| L(|x|)
$$

for all $0 \leq \lambda \leq \lambda_{0}, x \in X$, and almost all $0 \leq s, t \leq T$.

Assumptions (6.1) and (6.2) imply (cf. [11, Lemma 3.1]) that $\overline{D(A(t))}=D$ (constant), a.e. on $[0, T]$. Also recall (see Theorem 1 in [11]) that if (6.1) and $(6.2)$ hold, then the evolution equation

$$
\begin{gathered}
u^{\prime}(t)+A(t) u(t) \ni f(t), \quad 0<t<T \\
u(0)=u_{0}
\end{gathered}
$$

where $f \in L^{1}(0, T ; X)$ and $u_{0} \in D$, has a unique mild solution $u \in C([0, T] ; D)$. (The mild solution of (6.3) is obtained in essentially the same way as in the autonomous case, as a uniform limit of approximate solutions; see [11] for details.) Moreover, (cf. [11, Theorem 3]), if $u$ and $\widehat{u}$ are mild solutions of $(6.3)$ corresponding to $\left(f, u_{0}\right)$ and $\left(\widehat{f}, \widehat{u}_{0}\right)$ respectively, then $(2.2)$ holds.

We now consider the time-dependent Cauchy problem

$$
\begin{gathered}
u^{\prime}(t)+A(t) u(t)+G(u)(t) \ni 0,0<t<T \\
u(0)=g(u)
\end{gathered}
$$

where $G: C([0, T] ; D) \rightarrow L^{1}(0, T ; X)$ and $g: C([0, T] ; D) \rightarrow D$ satisfy $(H 2)$ and $(H 3)$ with $D$ in place of $\overline{D(A)}$. As in Section 3 , we say that $u \in C([0, T] ; D)$ is mild solution of (6.4) if it is a mild solution to (6.3) with $f=-G(u)$ and $u_{0}=g(u)$. By adapting the proof of Theorem 3.3 we arrive at the following result:

Theorem 6.1: Let (6.1), (6.2), (H2), (H3) (with D instead of $\overline{D(A))}$, and (3.1) be satisfied. Then problem (6.4) has a unique mild solution.

If $X$ is a Hilbert space and $A(t)$ are cyclically maximal monotone operators in $X$, we may allow $\overline{D(A(t))}$ to vary in time. In this case we obtain a strong solution to 
(6.1), that is a function $u \in W^{1,1}(0, T ; X)$ satisfying $(6.4)_{1}$, a.e. on $(0, T)$, and $(6.4)_{2}$. Following [15], we specifically assume that $A(t)=\partial \varphi^{t}$, where $\left\{\varphi^{t}, 0 \leq t \leq T\right\}$ is a family of proper convex, l.s.c. functions from $X$ into $(-\infty,+\infty]$ satisfying:

For each $r>0$, there are functions $c_{r} \in W^{1,2}(0, T), d_{r} \in W^{1,1}(0, T)$ with the property that for all $s, t \in[0, T], s \leq t$, and $z \in D\left(\varphi^{s}\right)$ with $|z| \leq r$, there exists $z_{1} \in D\left(\varphi^{t}\right)$ such that

and

$$
\left|z_{1}-z\right| \leq\left|c_{r}(t)-c_{r}(s)\right|\left(1+\left|\varphi^{s}(z)\right|^{1 / 2}\right)
$$

$$
\varphi^{t}\left(z_{1}\right)-\varphi^{s}(z) \leq\left|d_{r}(t)-d_{r}(s)\right|\left(1+\left|\varphi^{s}(z)\right|\right) .
$$

Theorem 6.2: Assume that $X$ is a Hilbert space and $A(t)=\partial \varphi^{t}, 0 \leq t \leq T$, with $\varphi^{t}$ satisfying (6.5). Let (H2), (H3) (with $X$ in place of $\overline{D(A))}$, and $(3.1)$ hold. If also $R(G) \subset L^{2}(0, T ; X)$ and $R(g) \subset D\left(\varphi^{0}\right)$, then (6.4) has a unique strong solution $u \in W^{1,2}(0, T ; X)$.

Proof: By Theorems 1.1.2 and 1.5.1 in [15], it follows that under the assumptions of Theorem 6.2 the initial value problem

$$
\begin{gathered}
u^{\prime}(t)+\partial \varphi^{t}(u(t))+G(v)(t) \ni 0,0<t<T \\
u(0)=g(v)
\end{gathered}
$$

has a unique solution $u \in W^{1,2}(0, T ; X)$ for each $v \in C([0, T] ; X)$. Define $J: C([0, T]$; $X) \rightarrow C([0, T] ; X)$ by $J v=u$, where $u$ solves (6.6). Use Theorem 1.1.1 in [15] in conjunction with $(H 2)$ and $(H 3)$ to obtain

$$
\|J v-J w\|_{C([0, T] ; X)} \leq(m+M)\|v-w\|_{C([0, T] ; X)}
$$

for any $v, w \in C([0, T] ; X)$. By (3.1), this shows that $J$ is a strict contraction on $C([0, T] ; X)$ and establishes the result.

\section{An Example}

An $\Omega$ be a bounded domain in $\mathbb{R}^{N}$ with smooth boundary $\Gamma$. We consider the initialboundary value problem

$$
\begin{gathered}
u_{t}(t, x)-\Delta_{x} u(t, x)+f_{1}(t, u(t, x))+\int_{0}^{T} a(t, s) f_{2}(s, u(s, x)) d s=0 \\
\text { a.e. on }(0, T) \times \Omega \\
-\frac{\partial u}{\partial \nu}(t, x) \in \beta(u(t, x)), \text { a.e. on }(0, T) \times \Gamma \\
u(0, x)=\sum_{i=1}^{n} h_{i}(x) u\left(t_{i}, x\right)+\int_{0}^{T} b(s) f_{3}(s, u(s, x)) d x, \text { a.e. on } \Omega
\end{gathered}
$$

where $\partial / \partial \nu$ denotes the outward normal derivative, and $0<t_{1}<\ldots<t_{n} \leq T$ are 
fixed. The following conditions will be imposed on the data of $(7.1): f_{i}:[0, T] \times \mathbb{R} \rightarrow \mathbb{R}$ $(i=1,2,3)$ are Carathéodory type functions such that

$$
f_{i}(\cdot, 0) \in L^{2}(0, T) \text { and }\left|f_{i}(t, x)-f_{i}(t, y)\right| \leq k_{i}(t)|x-y|, \text { for all } x, y \in \mathbb{R}
$$

and a.a. $t \in(0, T)$, with $k_{i} \in L^{2}(0, T)$,

$\beta$ is a maximal monotone graph in $\mathbb{R} \times \mathbb{R}$ with $0 \in \beta(0)$,

$$
a \in L^{2}\left((0, T)^{2}\right), \quad b \in L^{2}(0, T), h_{i} \in L^{\infty}(\Omega) \quad(i=1, \ldots, n) .
$$

Let $X=L^{2}(\Omega)$ and set

$$
A=-\Delta, D(A)=\left\{u \in H^{2}(\Omega):-\frac{\partial u}{\partial \nu} \in \beta(u), \text { a.e. on } \Gamma\right\} .
$$

By [3, Theorem 12], $A$ is cyclically maximal monotone in $X$; moreover, by (7.3), $\overline{D(A)}=X$. Next define $G: C([0, T] ; X) \rightarrow L^{2}(0, T ; X)$ and $g: C([0, T] ; X) \rightarrow X$ by

and, respectively,

$$
G(u)(t, \cdot)=f_{1}(t, u(t, \cdot))+\int_{0}^{T} a(t, s) f_{2}(s, u(s, \cdot)) d s
$$

$$
g(u)(\cdot)=\sum_{i=1}^{n} h_{i}(\cdot) u\left(t_{i}, \cdot\right)+\int_{0}^{T} b(s) f_{3}(u(s, \cdot)) d s .
$$

In view of (7.2) and (7.4) it is easily seen that $G$ and $g$ satisfy $(H 2)$ and $(H 3)$ with

$$
\begin{gathered}
M=\int_{0}^{T}\left|k_{1}(t)\right| d t+\int_{0}^{T} \int_{0}^{T}|a(t, s)|\left|k_{2}(s)\right| d s d t \\
m=\sum_{i=1}^{n}\left\|h_{i}\right\|_{L^{\infty}(\Omega)}+\int_{0}^{T}\left|b(s) k_{3}(s)\right| d s
\end{gathered}
$$

Hence, if we require that

$$
\left\|k_{1}\right\|_{L^{1}(0, T)}+\left\|a k_{2}\right\|_{L^{1}\left((0, T)^{2}\right)}+\left\|b k_{3}\right\|_{L^{1}(0, T)}+\sum_{i=1}^{n}\left\|h_{i}\right\|_{L^{\infty}(\Omega)}<1
$$

then (3.1) holds.

It is now obvious that (7.1) can be rewritten in the abstract form (1.1) in $X=L^{2}(\Omega)$ with $A, G$, and $g$ given by (7.5), (7.6), and (7.7) respectively. A direct application of Theorem 3.7 (first part) yields.

Theorem 7.1: If (7.1)-(7.4) and (7.8) are satisfied, then problem (7.1) has a unique solution $u \in C\left([0, T] ; L^{2}(\Omega)\right) \cap W^{1,2}\left(\varepsilon, T ; L^{2}(\Omega)\right) \quad(\forall \varepsilon \in(0, T))$, with $u(t, \cdot) \in$ $H^{2}(\Omega)$, a.e. on $(0, T)$. 


\section{References}

[1] Baillon, J.B. and Clément, P., Ergodic theorems for nonlinear Volterra equations in Hil-bert space, Nonlinear Anal. 5 (1981), 789-801.

[2] Balachandran, K. and Ilamaran, S., Existence and uniqueness of mild and strong solutions of a semilinear evolution equation with nonlocal condition, Indian J. Pure Appl. Math. 25 (1994), 411-418.

[3] Brézis, H., Monotonicity methods in Hilbert spaces and some applications to nonlinear partial differential equations, In: Contributions to Nonlinear Functional Analysis (ed. by E. Zarantonello), Academic Press, New York (1971), 101-156.

[4] Brézis, H., Opérateurs Maximaux Monotones et Semigroupes de Contractions dans les Espaces de Hilbert, North Holland, Amsterdam 1973.

[5] Byszewski, L., Theorems about the existence and uniqueness of solutions of a semilinear evolution nonlocal Cauchy problem, J. Math. Anal. Appl. 162 (1991), 494-505.

[6] Byszewski, L., Uniqueness criterion for solution of abstract nonlocal Cauchy problem, J. Appl. Math. Stoch. Anal. 6 (1993), 49-54.

[7] Byszewski, L., Existence and uniqueness of solutions of semilinear evolution nonlocal Cauchy problem, Zeszyty Nauk. Politech. Rzeszowskiej Mat. Fiz. 18 (1993), 109-112.

[8] Crandall, M.G., Nonlinear semigroups and evolution governed by accretive operators, In: Nonlinear Functional Analysis and its Applications (ed. by F.E. Browder), Proceedings of Symposia in Pure Math 45:1, AMS, Providence, RI (1986), 305-338.

[9] Crandall, M.G. and Nohel, J.A., An abstract functional differential equation and a related nonlinear Volterra equation, Israel J. Math. 29 (1978), 313-328.

[10] Deng, K., Exponential decay of solutions of semilinear parabolic equations with nonlocal initial conditions, J. Math. Anal. Appl. 179 (1993), 630-637.

[11] Evans, L.C., Nonlinear evolution equations in Banach spaces, Israel J. Math. 11 (1972), 57-94.

[12] Hulbert, D.S. and Reich, S., Asymptotic behavior of solutions to nonlinear Volterra integral equations, J. Math. Anal. Appl. 104 (1984), 155-172.

[13] Jackson, D., Existence and uniqueness of solutions to semilinear nonlocal parabolic equations, J. Math. Anal. Appl. 172 (1993), 256-265.

[14] Kato, N., Kobayasi, K. and Miyadera, I., On the asymptotic behavior of evolution equations associated with nonlinear Volterra equations, Nonlinear Anal. 9 (1985), 419-430.

[15] Kenmochi, N., Solvability of nonlinear evolution equations with time-dependent constraints and applications, Bull. Fac. Ed. Chiba Univ. 30 (1981), 1-87.

[16] Lin, Y, Analytical and numerical solutions for a class of nonlocal nonlinear parabolic differential equations, SIAM J. Math. Anal. 25 (1994), 1577-1596.

[17] Lin, Y. and Liu, J.H., Semilinear integrodifferential equations with nonlocal Cauchy problem, Nonlinear Anal. 26 (1996), 1023-1033.

[18] Nohel, J.A. and Shea, D.F., Frequency domain methods for Volterra equations, Adv. Math. 22 (1976), 278-304. 


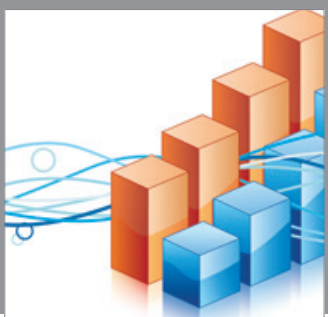

Advances in

Operations Research

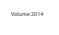

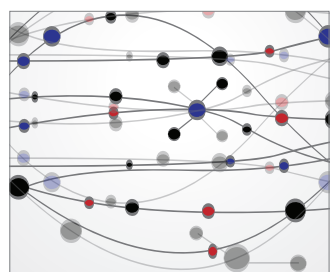

\section{The Scientific} World Journal
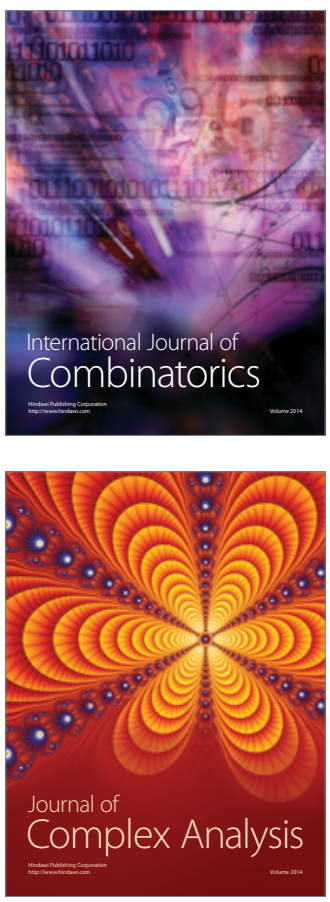

International Journal of

Mathematics and

Mathematical

Sciences
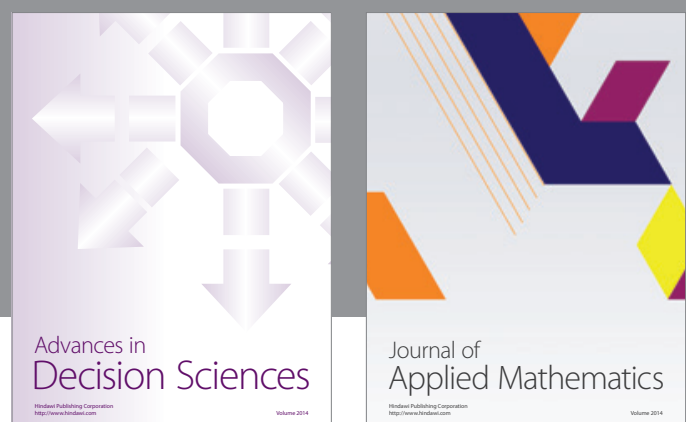

Journal of

Applied Mathematics
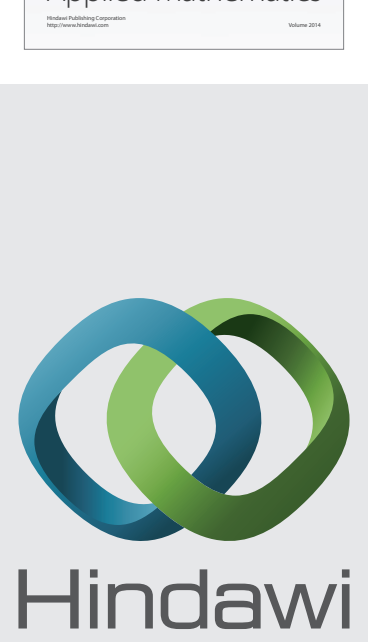

Submit your manuscripts at http://www.hindawi.com
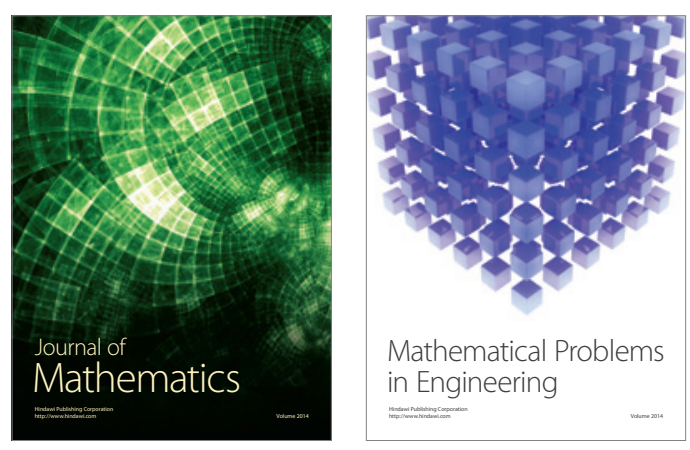

Mathematical Problems in Engineering
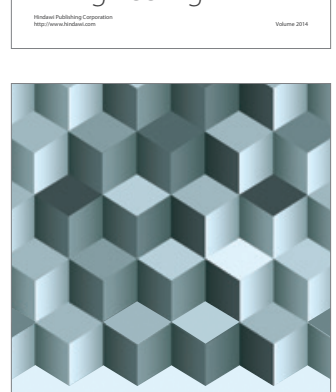

Journal of

Function Spaces
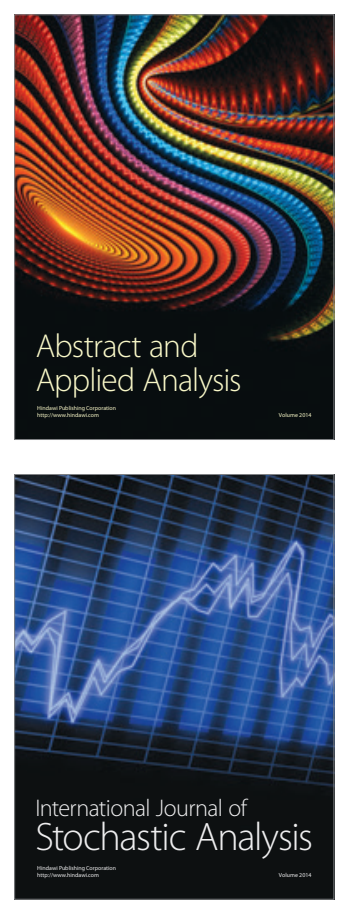

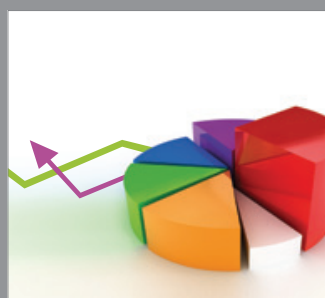

ournal of

Probability and Statistics

Promensencen
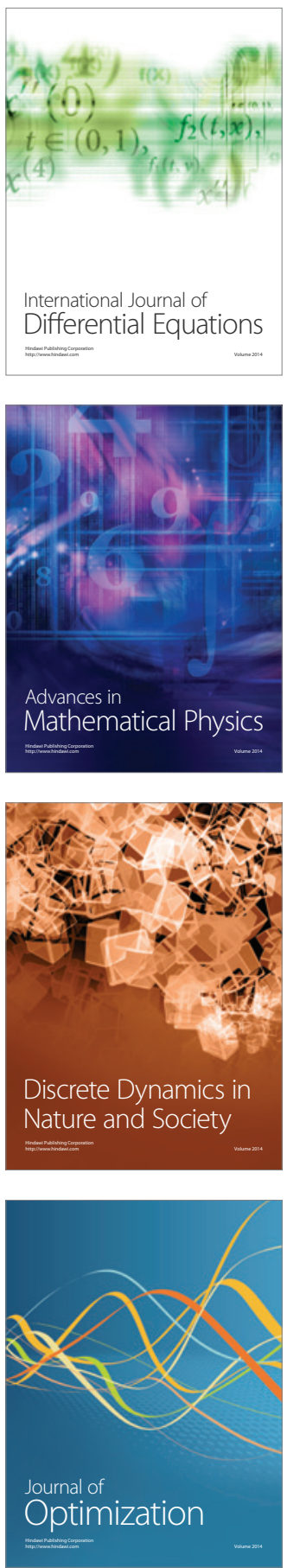